\title{
Divergência e Distribuição Industrial no Rio Grande do Sul (1985-2003)
}

Igor Alexandre Clemente de Morais*

Vanessa Frainer"*

Resumo: Este artigo tem como objetivo abordar os aspectos de Divergência e de distribuição do porte das empresas no Rio Grande do Sul, a partir de duas metodologias. A comparação da estrutura industrial entre diferentes regiões do Estado, é feita com o índice de Divergência regional proposto por Krugman (1997). Os resultados comprovam que, apesar da tendência de redução das disparidades na estrutura industrial, esse processo ainda é lento e preserva a existência de forte Divergência. Em relação à distribuição do porte das indústrias, as diversas funções de densidade estimadas indicam uma distribuição não gaussiana para o emprego. Além disso, os resultados para o modelo linear proposto por Zipf(1949) sinalizam a existência de disparidades na oportunidade de crescimento entre os portes de empresas. O modelo não-linear proposto por Rosen et al. (1980), indica a existência de concavidade, revelando que as empresas de porte intermediário, as pequenas e médias, representam a dinâmica do emprego do setor no período de análise.

Palavras Chave: Concentração Industrial, Economia Regional e Urbana, Lei de Zipf, Índice de Divergência.

\begin{abstract}
This article aims to address the issues of Divergence and of distribution of the size of companies in Rio Grande do Sul uaing two methodologies. A comparison of industrial structure between different regions of the state, is made with the index of regional divergence proposed by Krugman (1997). The results show that, despite the trend of reducing disparities in industrial structure, this process is still slow and preserves the existence of strong Divergence. In relation to the size distribution of industries, the various functions of the estimated density indicates a non Gaussian distribution for employment. In addition, the results for the linear model proposed by Zipf (1949) indicate the existence of disparities in opportunity for growth between the sizes of companies. The nonlinear model proposed by Rosen et al. (1980), indicates a concavity function, revealing that the size of intermediary companies, small and medium, representing the dynamics of employment in the sector in the period of analysis.
\end{abstract}

Keywords: Industrial Concentration, Regional....

Jel Classification: C2, N9, R1.

\footnotetext{
* Professor do Programa de Pós-Graduação em Economia da Unisinos e economista chefe da FIERGS. E-mail: igor@fiergs.org.br.

** FIERGS. E-mail: vfrainer@fiergs.org.br.

Recebido em 08 de março de 2007. Aceito em 02 de janeiro de 2008.
} 


\section{Introdução}

De uma forma geral, o estudo da economia regional pode ser dividido a partir de duas correntes que exercem forte influência no desenvolvimento teórico recente, a teoria clássica da localização e as teorias do desenvolvimento regional com ênfase nos fatores de aglomeração.

Os primeiros estudos na área de economia regional consideravam apenas a relação locacional das decisões de produção que eram influenciadas pelos custos de transporte. A seguir, a teoria do desenvolvimento regional incorporou a influência dos fatores de aglomeração, postulando que estes geram externalidades e ganhos de escala para a região e as firmas. No caso dessas, as economias de escala surgem devido a formação de clusters de firmas em uma determinada cidade ou região. Tal fato contribui para reduzir os custos de produção, atraindo mais fornecedores e gerando, assim, as economias de aglomeração. Como resultado das diferentes oportunidades de ocupação criadas nas cidades, as mesmas viram pólos de atração, crescem se desenvolvem e consolidam uma complexidade social urbana.

A nova teoria da geografia econômica resgata alguns aspectos relacionados ao tema, em especial quando se procura explicar as escolhas locacionais das firmas e seu processo de aglomeração ${ }^{1}$ nas áreas urbanas. Nesse caso, a abordagem da economia urbana é feita a partir do comportamento da firma, que determina a sua melhor localização considerando a influência de diferentes fatores.

Além da argumentação de que a concentração de empresas é um aspecto positivo para o crescimento econômico, Baumont et al. (2000) salientam que essa pode ser considerada como uma nova força de aglomeração. Mandariaga et al. (2005), usando dados de painel dinâmico da Argentina entre 1983 e 2002 e um filtro para corrigir a autocorrelação espacial, encontrou que a aglomeração de atividades deve ser considerada uma característica estrutural importante para explicar as diferenças no comportamento da taxa de crescimento e o estado estacionário das províncias daquele País. Além disso, o autor encontrou evidência da proximidade da força de trabalho para o crescimento econômico, com reflexos sobre a convergência da renda per capita.

1 Outras denominações desta linha de pensamento são a teorias neoclássicas de localização, ortodoxias teóricas, geometria germânica ou então eixo de teoria da localização. Para uma boa resenha da literatura de economia regional e urbana com a sistematização das diferentes linhas de pensamento, ver Cavalcante (2005). 
Aqui surgem os conceitos de distritos industriais e os ambientes inovadores, e a presença das externalidades de natureza tecnológica, a formação de redes de inovação e também a proposta de modelagem a partir dos retornos crescentes de escala, ver Krugman (1997).

Vale destacar que mesmo os fatores de aglomeração não sendo os únicos determinantes da escolha da localização de uma empresa, assim como apenas os custos de transporte e os efeitos spillovers da tecnologia, não se deve negligenciar a importância dos mesmos na decisão do local de produção. Argumenta-se que há uma série de aspectos que podem estar contribuindo para a formação de um cluster ou então de um parque industrial que apresente retornos crescentes de escala. Porém, grande parte dos estudos de impactos da integração econômica na concentração geográfica de indústrias, analisa dados dos E.U.A. e da União Européia, ver Ellison et al. (1997), Midelfart-Knarvik et al. (2000) e Brülhart (2001).

Algumas das características abordadas nessa literatura podem ser presenciadas em outros Países, a exemplo do Brasil, como salientado em Pacheco (1999), Resende et al. (2004) e Lage (2002), da mesma maneira que em uma análise regional, ver Suzigan et al. (2001, 2003).

No caso da economia do Rio Grande do Sul, o processo de crescimento e desenvolvimento histórico orientou a alocação de fatores de produção importantes, como a mão-de-obra. Nesse cenário, é possível constatar que a distribuição espacial dos empregos industriais não ocorreu de forma simétrica entre as diferentes regiões do Estado, havendo uma forte concentração de alguns gêneros que se especializaram na produção de determinados bens, ver Lautert (2005). Porém, a não aplicação de índices que possam medir essa Divergência industrial entre as regiões e como as mesmas se distribuem, deixa uma lacuna na literatura regional no Brasil.

Há diversos indicadores de economia regional que podem ser utilizados para medir concentração, localização, distribuição espacial e Divergência regional tanto de atividades econômicas como da população. ${ }^{2}$ Esse trabalho tem como objetivo principal avaliar dois aspectos relacionados à indústria do Rio Grande do Sul. Em primeiro lugar, a partir do modelo de Krugman (1997), mensurar o grau de Divergência regional, para dados de emprego industrial, comparado entre dois momentos no tempo, 1985 e 2003. Com o uso desse índice, será possível identificar a existência, ou não, de um processo de redução das disparidades regionais no período analisado.

2 Ver Haddad (1989) para uma resenha da literatura, e Krugman (1997) e Audretsch et al. (1996) e Suzigan et al. (2003), para uma aplicação de coeficientes de Gini locacionais... 
A segunda abordagem avalia a distribuição do tamanho das empresas industriais do Estado, a partir de dados de emprego, aplicando a lei de Zipf (1949) para caracterizar a existência ou não de um padrão na distribuição por porte. Ressalta-se que essa análise é útil para indicar o grau de concentração industrial presente na economia local, um resultado importante para as políticas industriais, ver Axtell (2001) para uma análise de dados de empresas dos E.U.A.

Além desta introdução, esse trabalho possui mais quatro outras seções. Na seção dois é feita uma breve descrição dos aspectos históricos do desenvolvimento e deslocamento da indústria entre as diferentes regiões do Estado, com o objetivo de apenas evidenciar essas características. A seguir, na seção três, são explicadas as metodologias utilizadas para mensurar esse processo, fundamentalmente a proposta de Krugman (1997) para caracterizar as disparidades regionais e a aplicação da lei de Zipf para a distribuição espacial. Na seção quatro, há uma breve descrição dos dados utilizados e a discussão dos resultados encontrados. Por fim, a seção cinco conclui o trabalho e propõe estudos futuros sobre o tema.

\section{Aspectos históricos do desenvolvimento da indústria gaúcha}

No período entre as duas guerras mundiais, o Rio Grande do Sul era visto como o celeiro do Brasil e ainda não estava muito aquém da economia de São Paulo. Os elevados custos de transporte, aliado ao significativo fluxo de imigrantes italianos e alemães com uma forte tradição cultural empreendedora, contribuiu para permitir o desenvolvimento da região e consolidar uma estrutura produtiva altamente diversificada. Essa era baseada em empresas familiares de pequeno porte e com uma concentração significativa nos setores de metalurgia, couros e calçados, têxteis, vestuário, bebidas e de alimentos, ver Fonseca (1980, 1984, 1992) e Lautert (2005).

Na década de 50, até meados dos anos 60, o ciclo de investimentos externos que havia trazido novos setores industriais importantes para a região Sudeste e, especialmente, para São Paulo - tais como as montadoras de veículos - fora virtualmente completado, com o Rio Grande do Sul à margem desse processo. ${ }^{3}$ A significativa redução dos custos de transporte presenciado na economia brasileira a partir

Monastério (2004) aplicou a lei de Zipf para o Rio Grande do Sul.

3 A política de substituição de importações foi um fator importante que concorreu para o aumento dos desequilíbrios regionais. Para uma discussão sobre esse tema, ver Fonseca (2003). 
da década de 60, tornou possível às empresas beneficiarem-se de ganhos de escala a partir da concentração da produção na região que detivesse uma maior concentração populacional e o maior potencial de mercado, ver Lage (2002) e Pacheco (1999).

Neste processo de integração espacial, em que perdem as atividades e os mercados locais, a economia do Rio Grande do Sul, à semelhança do que ocorria nas demais regiões periféricas do Brasil, sofreu abalos significativos. Progressivamente, o Estado foi atingido pela maior competitividade das empresas localizadas na região dominante do nosso sistema financeiro local, como os setores produtores de bebidas, têxteis e vestuário, material elétrico e, especialmente alimentos.

A história subseqüente da economia gaúcha a partir da década de 60 teria sido de contínuo declínio não fosse a ocorrência de três fatos externos de grande importância local. Esses envolveram a intervenção governamental na forma de políticas industriais, definidas em seu sentido econômico amplo, ou seja, como intervenções seletivas em setores da economia, inclusive a agricultura, com o objetivo de impulsionar o crescimento.

O primeiro desses consistiu em importantes investimentos de infra-estrutura realizados pelo Governo Federal no Rio Grande do Sul. Assim, após a implantação da Refinaria Alberto Pasqualini ainda no início dos anos 60, sucederam-se os investimentos no porto de Rio Grande, Aços Finos Piratini, a malha de rodovias federais e, finalmente, o pólo petroquímico de Triunfo. Tais investimentos foram extremamente importantes para a reestruturação do setor industrial e o relançamento da atividade exportadora local. Além disso, estava em curso um processo de transformação na distribuição espacial da indústria no Estado, ver Lautert (2005) Vieira et al. (1993), destaca que esse processo também foi influenciado pelos investimentos, feito pelo Governo do Estado em infra-estrutura, para interligar as regiões interioranas.

O segundo fato teve a ver com o forte estímulo do governo federal à produção de grãos - especialmente soja e trigo - e de outras culturas para a exportação em substituição à pecuária tradicional. $\mathrm{O}$ crescimento da produtividade na área das lavouras além da elevação do preço da terra permitiu uma substituição mais rápida da pecuária extensiva por uma atividade mais intensiva, calcada na absorção de novas tecnologias. Dessa forma, o setor agropecuário, que entrara em estagnação com o declínio dos frigoríficos e da indústria de carnes, voltou a ter, novamente, um papel importante como fonte de crescimento da economia gaúcha. Ver Alonso et al. (1994) e Alves 
(2006), para uma discussão sobre a dinâmica de crescimento da agropecuária no Estado e Stülp (2006), para uma abordagem sobre a produtividade da mão-de-obra no setor.

O terceiro fato decorreu também de uma política do governo federal no sentido de criar, através de incentivos fiscais e de crédito, uma forte base exportadora no País, ver Portugal (1994) para uma análise das políticas de comércio exterior implementadas entre 1947 e 1988. Esta forma de política industrial veio justamente ao encontro da economia gaúcha, cuja tradição exportadora já estava presente no século passado, sendo, pois, um traço cultural importante na região.

Na década de 1980, o investimento público que havia se constituído em uma importante fonte de crescimento ao longo dos anos 70 , foi substancialmente reduzido, em face das dificuldades fiscais crescentes do Estado nacional. A crise da dívida externa a partir de 1982, os fracassos dos planos de estabilização da economia desde 1986 e a moratória externa de 1987, determinaram o padrão de instabilidade e estagnação econômica no Brasil que haveria de atravessar toda a década seguinte, ver Giambiagi et al. (2004). A economia gaúcha não estaria imune a esse cenário, que contribuiu para modificar a configuração da indústria local, ver Lautert (2005).

Os diversos elementos externos acima citados, bem como a própria característica histórica da formação industrial do Estado, são elementos importantes a explicar a dinâmica recente do setor. Além disso, entender e mensurar o processo de concentração da indústria, a partir de sua distribuição, bem como o grau de Divergência que o mesmo ocasiona nas diferentes regiões do Rio Grande do Sul, são aspectos que podem ajudar a explicar esses fatores. $\mathrm{Na}$ próxima seção são descritos os indicadores a serem utilizados nesse trabalho para quantificar essas características.

\section{Metodologia}

Essa seção é dividida em duas partes. Na primeira descreve-se o indicador de Divergência proposto por Krugman (1997), útil para indicar o grau das disparidades regionais. A seguir, é explicada a metodologia para medir a distribuição das empresas.

A proposta de Krugman (1997) é dada pela relação (1):

$$
I D=\sum_{i=1}^{n}\left|s_{i}-s_{i}^{*}\right|
$$


com $i$ representando as diferentes indústrias, $S_{i}$ a participação do emprego do setor $i$ no total do emprego na indústria em alguma dada região, e $s_{i} *$ a mesma relação porém, para uma outra região. ${ }^{4}$ Como pode ser visto, o índice de Divergência faz uma comparação da estrutura setorial entre diferentes regiões, ou seja, a importância de um determinado setor em uma dada região com o mesmo setor em outra região.

O resultado do índice fica no intervalo $0 \leq I D \leq 2$, de tal forma que quanto maior for $I D$, mais distinta deverá ser a estrutura industrial entre as duas regióes analisadas. Sendo assim, o valor de $I D=0$ ocorre quando as duas regiões possuírem a mesma estrutura industrial. Por outro lado, se essa estrutura for completamente diferente, então tem-se que $I D=2$

Krugman (1997) aplicou este índice para dados de emprego de países europeus e regiões dos EUA para comparar o grau de Divergência dentre elas. Os dados utilizados tinham grau de abertura de quatro dígitos para o emprego industrial e, a conclusão é que as regiões nos EUA aparentam maior Divergência que nos países europeus.

Apesar da aplicabilidade e simplicidade do modelo proposto, o mesmo não está livre de críticas. Em primeiro lugar que sua formulação não permite captar, por exemplo, questões específicas que possam estar gerando a formação de maior Divergência entre as regiões. Além disso, não modela diferenças de estrutura de mercado entre os setores, ver Marques (2001) para uma crítica ao modelo.

Além da identificação do grau de Divergência que existe entre as regiões, uma outra parte da literatura propõe analisar a distribuição espacial, como forma de verificar os indícios de formação de clusters ou aglomerações em centros importantes, ver Brakman et al. (1999 e 2005). Nesse caso, é testada a existência ou não de assimetria na distribuição da variável de controle, como por exemplo, o tamanho das cidades. Essas estimativas são feitas tendo como hipótese uma distribuição de pareto. ${ }^{5}$ É importante destacar que um caso especial dessa distribuição é conhecido na literatura como lei de Zipf, onde assume-se que o porte das cidades segue uma relação de linearidade, dada por 2:

$$
\ln (i)=\ln (a)+\zeta \ln (\text { tamanho })
$$

4 Para padronizar a referência, o indicador da equação 3.1 será aqui referido como índice de Divergência.

5 Essa é dada a partir de: $P(R>S)=\frac{a}{S^{\zeta}}$, sendo $R$ o tamanho das cidades, $S$ o número de habitantes, a uma constante, e z o expoente de Pareto, que pode assumir qualquer valor. 
com $i$ referindo-se à posição de cada cidade, ordenadas de forma decrescente $i=1,2, \ldots n$. Dessa forma, o coeficiente representa a população da maior cidade e, a variável tamanho, é o número de habitantes de cada uma. ${ }^{6}$

A expectativa para o parâmetro de inclinação, é que $\zeta \cong-1$, e se esse resultado se verificar, então existe uma regularidade que explica a relação de tamanho entre as cidades. A de maior porte tem o dobro do tamanho da segunda, um terço do porte da terceira cidade, e assim sucessivamente. ${ }^{7}$ Se $\zeta \neq-1$, esta característica não se verifica, sinalizando que existem cidades que estão apresentando diferentes oportunidades de crescimento. ${ }^{8}$

Apesar de simples, este modelo foi aplicado por diversos autores em vários países. Brakman et al. (1999) e Soo (2004), fazem comparações internacionais, Dobkins et al.(2000) para dados de crosssection dos municípios americanos e Black et al. (2003) com dados de painel.

Uma outra opção é testar a existência de uma relação não-linear. Isso pode ser feito a partir da adição de um termo quadrático ou cúbico na equação 2, tal como proposto por Rosen et al. (1980);

$$
\begin{aligned}
& \ln (i)=a+b \ln \left(S_{i}\right)+c \ln \left(S_{i}\right)^{2} \\
& \ln (i)=a^{\prime}+b^{\prime} \ln \left(S_{i}\right)+c^{\prime} \ln \left(S_{i}\right)^{2}+\gamma^{\prime} \ln \left(S_{i}\right)^{3}
\end{aligned}
$$

O parâmetro c está relacionado à curvatura da distribuição. Para $\mathrm{c}>0$, há convexidade e a distribuição contém um número menor de cidades de porte médio do que o previsto originalmente na lei de Zipf. Ou seja, existem poucas cidades de porte intermediário relativamente a cidades maiores ou menores. Assim, a maior cidade seria bem maior e as cidades menores seriam em número mais

Formalmente, a lei de Zipf é encontrada quando $\zeta=1$.

6 Gabaix et al. (2003), usam o procedimento de Monte Carlo para mostrar que esta regressão não é a mais apropriada para a análise da distribuição do tamanho das cidades, principalmente em amostras pequenas. Nishiyana et al. (2004) examinam as propriedades estatísticas do estimador de mínimos quadrados para a regressão da regra de rank-size do tamanho das cidades. Neste caso, os autores propõem a aplicação do método dos mínimos quadrados generalizados, devido o fato de que a variável explicada é heteroscedástica e autocorrelacionada.

7 Uma hipótese fundamental da Lei de Zipf é que, independente do tamanho das cidades ou a sua posição espacial, todas têm as mesmas potencialidades de crescimento. O debate sobre o tema pode ser visto nas publicações de Krugman (1996), Alperovich (1993) e Dobkins et al. (2000).

8 Para o caso em que $\zeta=0$ todas as cidades possuem o mesmo tamanho. E, se $\varsigma>-1$ então há indícios de aglomeração de cidades. 
acentuado do que o esperado na relação linear entre i e $S$, dada pela equação 2 .

De outra forma, para $\mathrm{c}<0$, o que representaria uma concavidade, há um número maior de cidades de tamanho intermediário, ver Black et al. (2003) para uma aplicação a dados da economia americana.

Gabaix et al. (2003) mostra que é possível verificar uma grande dispersão do coeficiente da lei de Zipf para diferentes estimativas, devendo este oscilar no intervalo $[0,85 ; 1,15]$. Apesar disso, o autor também salienta que um valor entre $[0,8 ; 1,2]$ estaria validando a aplicação do modelo. ${ }^{9}$

Além do uso para a investigação da distribuição das cidades, essa metodologia também pode ser aplicada na análise da distribuição do tamanho das empresas. Neste caso, é comum valer-se de dados de emprego, ordenando as empresas em ordem decrescente. Porém há poucos trabalhos na literatura internacional nesta linha, em especial os estudos de Okuyama et al. (1999) e Axtell (2001).

Neste trabalho é aplicada a metodologia de estimativa dos parâmetros da lei de Zipf para uma distribuição do tamanho das empresas, a partir da proposta linear e não-linear. Até o momento, na literatura nacional, esta metodologia não foi utilizada para dados de empresas, tanto no que diz respeito a Brasil quanto para o Rio Grande do Sul.

\section{Resultados empíricos}

Essa seção divide-se em duas partes. Na primeira, aplica-se a metodologia proposta por Krugman (1997) para caracterizar a Divergência industrial aos dados de emprego industrial do Rio Grande do Sul no período compreendido ${ }^{10}$ entre 1985 e 2003. Na segunda parte, o objetivo é aplicar a relação 2 e 3, a dados de emprego por empresa, para identificar a existência ou não de alguma regularidade na distribuição por porte.

\subsection{Divergência na indústria do Rio Grande do Sul}

Os dados utilizados nesta primeira parte foram extraídos da RAIS (Relação Anual de Informações Sociais) para 12 gêneros ${ }^{11}$ da indústria

\footnotetext{
9 Para uma comparação entre os diferentes resultados encontrados para o expoente de Pareto na literatura internacional consultar Delgado et al. (2004).

${ }^{10} \mathrm{O}$ ano de 1985 foi escolhido por ser o primeiro da série histórica de dados sobre o mercado de trabalho que estão disponíveis no Ministério do Trabalho e Emprego.

${ }^{11}$ São eles: Minerais não-metálicos; metalúrgica; mecânica; material elétrico e de comunicações;
} 
de transformação, em 1985 e 2003. É seguida a divisão do Estado de acordo com as sete Mesoregiões definidas pelo IBGE. Assim, utilizando a equação 1, para cada região foi calculada a diferença de participação do emprego por setor e, depois, obtido o índice de Divergência entre as regiões. Na tabela 1 estão as estimativas para o ano de 1985 e 2003.

Tabela 1. Índices de Divergência (diagonal inferior/1985 e diagonal superior/ 2003)

Fonte de dados brutos: RAIS/MTE. Cálculos feitos pelo autor

Como citado anteriormente, o índice de Divergência deve oscilar entre 0 e 2 , sendo que, quanto maior for seu valor, maior é a diferença de estrutura industrial presente entre as regiões. Na tabela 4.1, a diagonal inferior revela os resultados para o ano de 1985, onde verifica-se que a região Sudeste do Estado é a que apresenta os maiores valores, em especial quando se compara a sua estrutura industrial com a região Nordeste $(1,472)$ e a Metropolitana $(1,382)$. Uma outra região que também apresenta valores elevados para o índice, naquele ano, é a Sudoeste. A estrutura industrial destas duas regiões, quando comparadas, parecem ser similares, e o índice de Divergência tem valor de $(0,275)$, o menor dentre todos.

As estimativas indicam que, em meados da década de 80, existia uma forte divergência industrial no Rio Grande do Sul, com as regiões Sudeste e Sudoeste divergindo da estrutura predominante no eixo da região Metropolitana e Nordeste.

Naturalmente procura-se investigar se esta característica é histórica, como apontado em Lautert (2005) ou então um processo que se intensificou a partir das modificações de natureza macroeconômica que afetaram o Estado nos últimos anos. Para tanto, o índice

material de transporte; madeira e mobiliário; papel e papelão, editorial e gráfica; borracha, fumo, couros, peles, similares e indústrias diversas; química e produtos farmacêuticos, veterinários e perfumaria; têxtil, vestuário e artefatos de tecido; alimentos e bebidas; calçados. 
foi calculado para os dados de emprego no ano de 2003, e são mostrados na diagonal superior da tabela 1 .

Os dados revelam que ainda perdura uma enorme diferença industrial entre as sete regiões, ou seja, a indústria de transformação no Rio Grande do Sul continua altamente especializada e localizada. A Divergência entre a região Sudeste e Nordeste apesar de ainda continuar elevada, reduziu-se $(1,042)$ em relação ao ano de 1985 deixando a maior Divergência no Estado para as regiões Sudoeste e Metropolitana, cujo valor do índice é de (1,352).

Este resultado pode estar sendo explicado pelo fato de que a região Sudoeste respondia por apenas 1\% do emprego industrial do Estado em 2003, o menor valor dentre todas as regiões, ao passo que a região Metropolitana concentrava 52\% dos empregos totais no setor. Portanto, o índice dá suporte à constatação de duas questões. Primeiro que, em especial as regiões Sudoeste e Metropolitana, apresentam uma estrutura industrial bastante diferente. E, segundo, que essa Divergência perdura desde 1985.

As comparações do índice da região Sudoeste com outras regiões, também indica a existência de grande Divergência em 2003, como em relação à região Nordeste $(1,278)$ e também na comparação com a região Centro Oriental $(1,114)$. Além disso, os resultados para esse ano mostram-se um pouco maiores que os encontrados para o ano de 1985, sinalizando uma piora na disparidade regional.

Apesar de estes resultados indicarem que perdura na região Sudoeste uma estrutura industrial bastante distinta das demais regiões do Estado, a interpretação destes índices por gênero da indústria pode fornecer uma informação mais detalhada sobre como se comporta o grau de Divergência industrial.

Toma-se, por exemplo, o caso da região Metropolitana e Centro Oriental, justamente as que apresentam o menor índice de $\mathrm{Di}$ vergência em 2003, $(0,416)$. Esse resultado pode estar refletindo uma característica presente no setor nessas duas regiões, uma vez que ambas possuem um elevado grau de concentração dos empregos na indústria do calçado, $34 \%$ e $32 \%$ respectivamente e também de produtos alimentares e bebidas, $23 \%$ e 9,7\% respectivamente. Destaca-se que ambos os gêneros da indústria são intensivos no fator trabalho e que empregam juntos $40,2 \%$ da mão-de-obra na indústria do Estado.

Por outro lado, o índice de Divergência encontrado para a região Sudoeste e Sudeste, também é baixo, $(0,465)$ indicando uma estrutura industrial equivalente entre estas duas regiões. Porém, este resultado é verificado, pois estas são justamente as duas regiões de 
menor participação no emprego do setor no total do Rio Grande do Sul. Além disso, ambas as regiões são muito especializadas em uma única atividade industrial intensiva em trabalho. $O$ gênero de alimentos e bebidas representa $76 \%$ dos empregos na indústria da região Sudoeste, e 59\% na região Sudeste.

Apesar de estarem em dois extremos, com um índice de Divergência de $(1,352)$, algumas indústrias da região Metropolitana e Sudoeste possuem similaridades, como por exemplo, o gênero de minerais não-metálicos, material elétrico e de comunicação, material de transporte e também em têxtil, vestuário e artefatos de tecido. Mas, por outro lado, as diferenças são significativas em outras indústrias, como calçados, química e mecânica, estando estas quase que ausentes na região Sudoeste.

Comparando os resultados do ano de 1985 com 2003, pode-se ver que, de uma forma geral, ocorreu uma redução da divergência regional na indústria do Estado do Rio Grande do Sul no período, ao menos quando são utilizados os dados de emprego para construir o indicador. Este fato poderia ser explicado, em parte, pelo processo de urbanização. A aglomeração urbana em alguns centros médios pode ser apontada como fator de atração das indústrias que deslocam-se do eixo Porto Alegre-Caxias e, assim, geram uma menor concentração em determinada região, ver Monastério (2004) para uma aplicação da Lei de Zipf ao Rio Grande do Sul.

Além disso, a redução dos custos de transporte e de comunicação e os ganhos de escala que o desenvolvimento de cidades de porte médio experimentaram neste período, também podem ser citados como fatores que contribuíram para a redução destas disparidades. Ver Morais (2007) para uma análise dos fatores de impulso ao crescimento econômico e populacional no Estado.

Porém, mesmo diante de uma menor disparidade, a região Sudoeste está presente em cinco dos seis resultados que apresentaram crescimento da Divergência entre o ano de 1985 e 2003. Ou seja, no período esta região aumentou a sua distância em relação às demais no que diz respeito à atividade industrial. É importante também destacar que a região Sudoeste conseguiu reduzir a sua divergência estrutural neste setor apenas em relação à região Noroeste do Estado.

Por outro lado, uma das regiões que conseguiu obter grandes reduções na divergência industrial foi a Sudeste. Durante o período aqui analisado ocorreu um aumento da participação de diversos setores industriais na composição do PIB da região, como por exemplo, a madeira e o mobiliário, borracha, fumo, couros e peles e minerais nãometálicos, ver FEE (2003). Porém, para o mesmo período, a indústria de 
produtos alimentares teve redução da Divergência sem que, no entanto, esta perdesse a sua importância econômica para a região.

Portanto, a análise da Divergência regional a partir do indicador proposto por Krugman (1997), revela que, mesmo que de forma lenta, há evidências de redução das disparidades na estrutura industrial no Estado do Rio Grande do Sul. Como exceção, cita-se a região Sudoeste, que deve ser merecedora de uma maior atenção por parte das políticas públicas que tenham como objetivo a redução das desigualdades regionais, em especial no que diz respeito à indústria.

Apesar dos resultados do índice de Divergência revelarem algumas informações importantes sobre as características industriais, deixa a desejar no que diz respeito às variáveis que influenciaram este movimento no período considerado. De uma forma geral, fatores como a existência de um grande mercado consumidor, aglomeração populacional, os custos de transporte, as economias de escala com a aglomeração industrial, a disponibilidade de fatores de produção como mão-de-obra, terra e capital, os incentivos fiscais e o acesso a novas tecnologias, são citados na literatura como determinantes da localização industrial, ver Fujita et al. (1999).

Em particular, o tamanho do mercado consumidor pode ser medido pelo Produto Interno Bruto. Neste caso, a região Metropolitana destaca-se das demais por conter $45 \%$ do PIB do Rio Grande do Sul, ao passo que a região Sudoeste e a Centro Ocidental participam com apenas 3,5\% e 5,7\%, respectivamente. Além desta, a aglomeração populacional também é uma variável importante para dimensionar o mercado consumidor e, neste caso, a região Metropolitana participa com $43 \%$ do total do Estado, ao passo que a região Sudoeste apenas 7,3\% e a Centro Ocidental 5,2\%, ver FEE (2003).

Os custos de transporte podem ser medidos a partir da distância em quilômetros entre a principal cidade de cada região e o maior centro consumidor do Estado, a região Metropolitana. Sendo assim, a região Sudoeste, com a cidade de Uruguaiana, apresenta maiores custos relativamente à região Centro Ocidental, quando considerada a cidade de Santa Maria. ${ }^{12}$

Uma outra variável econômica que pode ser utilizada para explicar as economias de escala é a quantidade de indústrias em determinada região. A fundamentação por traz deste argumento é que,

12 Em 2000 a cidade de Uruguaiana contava com uma população de 126 mil pessoas e está $650 \mathrm{~km}$ distante de Porto Alegre, ao passo que a cidade de Santa Maria com 243 mil habitantes, é distante $286 \mathrm{~km}$ de Porto Alegre. Ver FEE (2001). 
quanto maior for o número de empresas em uma região, maiores serão os benefícios para que uma nova empresa ali se instale, uma vez que aumentam as chances de estar presente não apenas seus fornecedores como também uma outra indústria que é vista como sua demandante, ver Krugman (1997) e Baumont et al. (2000). Este é o aspecto de atração que a aglomeração exerce sobre a decisão de localização das empresas. Porém, como salientado em Marques (2001) e Fujita et al. (1999), os efeitos de deseconomias de aglomeração também podem atuar no sentido inverso, reduzindo a atratividade de novas indústrias.

Analisando os dados da RAIS sobre o número de estabelecimentos industriais no Rio Grande do Sul no ano de 2003, verifica-se que a região Centro Ocidental leva uma pequena vantagem sobre a região Sudoeste, na medida em que participa com cerca de 2,8\% do total dos estabelecimentos deste setor no Estado contra apenas $2 \%$ da região Sudoeste.

\subsection{A distribuição do porte das empresas}

A constatação da existência de uma grande divergência industrial entre as regiões do Rio Grande do Sul deve guardar uma estreita relação com o processo de distribuição do porte das empresas do setor, ver Axtell (2001) para uma discussão sobre as implicações de uma distribuição assimétrica no tamanho das empresas. Essa seção investiga esse aspecto a partir da identificação de uma função de distribuição que possa representar o processo gerador dos dados, ou seja, o tamanho das empresas medido pelo número de empregados. Além disso, também é verificado se essa distribuição apresenta alguma regularidade, como indicado pela Lei de Zipf.

A fonte de dados são as informações individuais do emprego médio industrial dos últimos 12 meses terminados em agosto ${ }^{13}$ de 2005, perfazendo um total de 3.382 empresas. A tabela 4.2 mostra um resumo estatístico da amostra.

A soma de todos os empregados é de 398 mil, o que representa cerca de $70 \%$ do total dos empregos formais no setor para o ano em questão, comparando com os dados da RAIS. A divisão por porte, revela a presença de uma grande quantidade de empresas micro e pequena, característica esta que pode contribuir para a existência de curtose e assimetria na função de distribuição. ${ }^{14}$

${ }^{13}$ Dados obtidos junto ao CIAF/FIERGS.

${ }^{14} \mathrm{O}$ teste de Jarque-Bera, 398.168, com p-valor $=0,000$, rejeita a hipótese nula de distribuição normal. 
Tabela 2. Resumo estatístico dos dados de emprego por empresa

Fonte de dados brutos: CIAF/FIERGS

A partir desses dados, foram estimadas diversas funções de distribuição, mostradas no conjunto de gráficos 4.1. Todas as distribuições estimadas mostram características interessantes. Em primeiro lugar a existência de curtose, comprovada pelo teste e que também pode ser vista a partir da curva achatada, fato atribuído à maior incidência de empresas de tamanho intermediário. Em segundo lugar, a assimetria positiva, também comprovada pelo teste, e que pode estar ocorrendo devido a pouca presença de empresas de porte grande. E, em terceiro lugar a boa aderência entre as curvas estimadas. ${ }^{15}$

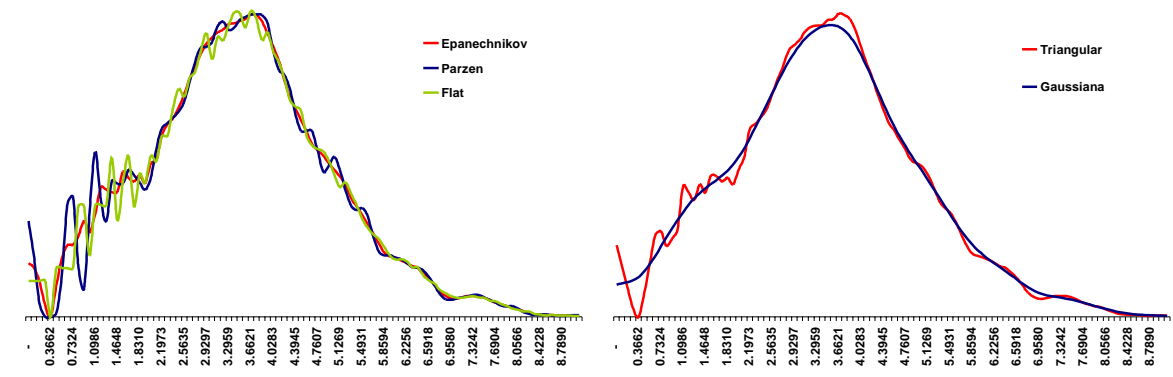

Gráfico 1. Funções de densidade para os empregos industriais - Rio Grande do Sul Fonte de dados brutos: CIAF/FIERGS

15 Para estas estimativas foi utilizado o pacote estatístico RATS. Formalmente, a estimativa da densidade de Kernel de uma série de dados $X$ no ponto $x$ é dada por: $f_{(x)}=\frac{1}{n h} \sum_{i=1}^{n} K\left(\frac{x-X_{i}}{h}\right)$, sendo que $n$ é o número de observações, $h$ é o fator de alisamento da série e $k$ é a função de peso de Kernel que, no presente estudo, é a epanechnikov. 
Tais resultados indicam que a relação entre o porte das empresas industriais, não é linear, e pode ser testado a partir da estimativa das equações 2 e 3 Nesse caso, o primeiro passo é ordenar as empresas em ordem decrescente do número de empregados, e representá-los a partir da lei de Zipf, como mostra o gráfico 2.

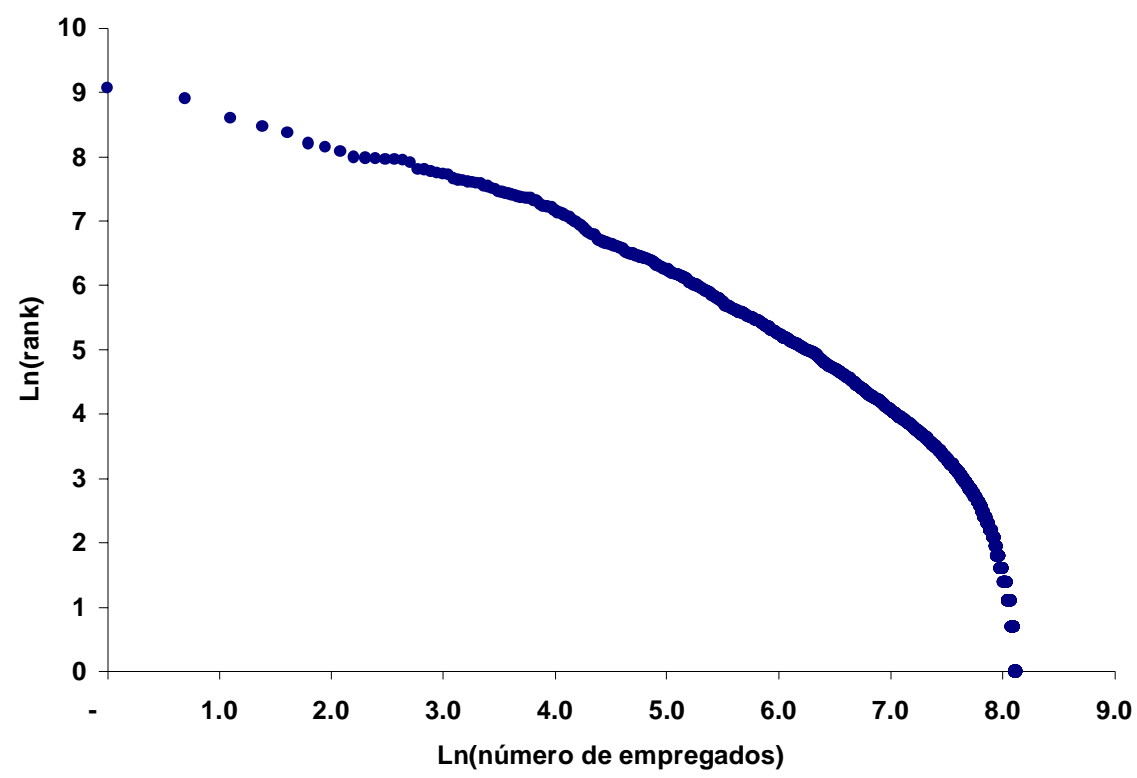

Gráfico 2. Lei de Zipf para as indústrias gaúchas

A relação entre tamanho da empresa, dado por ln(número de empregados) e a ordem, ln(rank), também sinaliza para uma relação não-linear. Iniciando pela investigação linear, a expectativa era que o coeficiente que mede esta relação fosse de ordem um. Esse seria o caso especial da distribuição de Pareto, conhecido como a Lei de Zipf. Porém, este não parece ser o processo verificado e, provavelmente, o coeficiente de Pareto terá um valor menor do que a unidade.

De fato, a estimativa para a equação 2 , a partir do método de mínimos quadrados ordinários gera um resultado para o coeficiente de Pareto menor do que a unidade, como esperado, $b=-0,589$. Esse valor indica que as empresas industriais no Rio Grande do Sul não experimentam as mesmas oportunidades de crescimento de acordo com o porte, pelo menos no que diz respeito à geração de empregos. Os testes feitos para os resíduos não indicam a presença de autocorrelação ou heteroscedasticidade. Porém, os mesmos não possuem distribuição normal. 


$$
\ln (i)=\underset{(0,016)}{9,157-0,589 \ln \left(S_{i}\right)}
$$

Schwarz $=0,92$

Jarque-Bera $=19.219$

White $=1.625$

D-Watson $=0,001$
Akaike $=0,91$

$\operatorname{ARCH}(1)=243.590$

Breusch-Godfrey $=53.587$

$\mathrm{R}^{2}$ ajustado $=0,85$

As estimativas não-lineares, feitas a partir da equação 3, revelam a existência de um valor negativo para o coeficiente $\mathrm{c}$, indicando a presença de concavidade para a distribuição do tamanho das empresas industriais no Rio Grande do Sul, ilustrado no gráfico 3 a partir dos resultados estimados para cada nível de emprego.

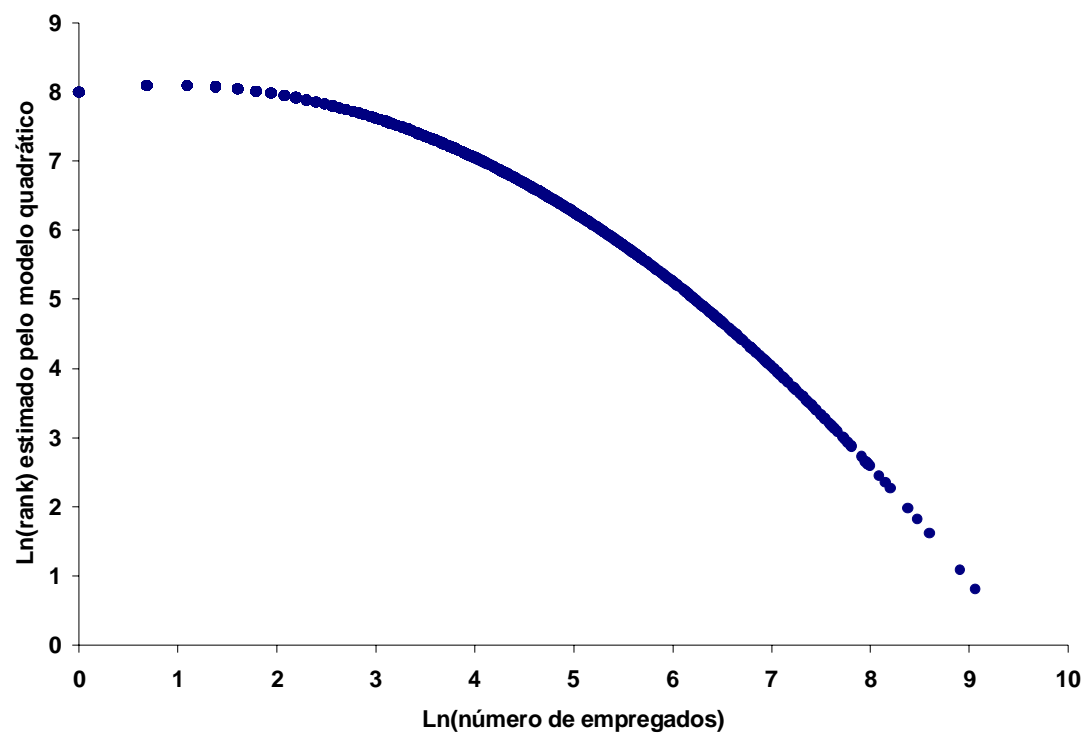

Gráfico 3. Lei de Zipf para as indústrias gaúchas - modelo não-linear

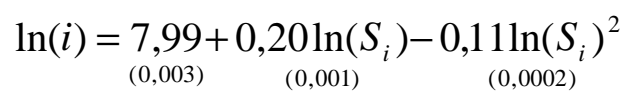




$\begin{array}{ll}\text { Schwarz }=-3,31 & \text { Akaike }=-3,32 \\ \text { Jarque-Bera }=256.344 & \text { ARCH }(1)=5.470 \\ \text { White }=881 & \text { Breusch-Godfrey }=13.832 \\ \text { D-Watson }=0,046 & \text { R2ajustado }=0,99\end{array}$

Esses resultados sinalizam que a evolução do emprego na indústria não foi acompanhada pela concentração do mesmo nas maiores empresas, e nem ocorreu uma proliferação de empresas de porte micro. Na verdade, são as empresas de porte intermediário, pequenas e médias, que podem estar representando a dinâmica do emprego no setor no período de análise. Os testes feitos para os resíduos não indicam a presença de autocorrelação ou heteroscedasticidade, mas os mesmos não possuem distribuição normal.

Apesar de ter sido encontrado que o modelo não-linear produziu um valor com sinal diferente para o termo que representa a linearidade, $b=0,20$, este parece ser um resultado comum na literatura, tendo sido também observado em Soo (2004) e em Rosen et al. (1980). ${ }^{16}$

\section{Conclusão}

Este trabalho aplicou a proposta de Krugman (1997) para caracterizar a Divergência da indústria entre as sete Mesoregiões do Rio Grande do Sul definidas pelo IBGE, no ano de 1985 e 2003. Além disso, foi estimada uma relação linear e outra não-linear com o objetivo de verificar a existência de uma regularidade na distribuição do porte de empresas na indústria do Estado.

No primeiro caso, os resultados mostram que, apesar de se caracterizar uma redução das divergências regionais em relação ao setor industrial, este processo mostra-se lento, e ainda preserva características distintas. Em especial, as regiões Sudeste e Sudoeste do Estado revelam uma elevada disparidade em relação às demais.

De acordo com Fujita et al. (1999), as firmas avaliam os mesmos tipos de fatores de produção de forma distinta, procurando se localizar em regiões onde os seus insumos mais importantes estão disponíveis. Dessa forma, alguns fatores específicos, como disponibilidade

${ }^{16}$ Enquanto que na equação linear o coeficiente $b$ mede a inclinação da melhor reta, no modelo quadrático o termo linear fornece a localização dos pontos de máximo e mínimo desta melhor reta. 
de mão-de-obra, infra-estrutura logística e capital humano podem ser vistos como determinantes nesse processo lento de redução das disparidades regionais na indústria do Estado.

O segundo objeto de investigação foi a distribuição do porte das empresas. As estimativas não indicam a existência de uma distribuição normal para os empregos, sendo possível identificar assimetria e curtose nos dados. Esses aspectos são esperados na medida em que há poucas grandes empresas e uma grande quantidade de micro e pequenas.

Tal resultado também pode ser comprovado a partir de estimativas lineares e não-lineares da relação do porte das empresas. Neste caso, as estimativas para o modelo linear, revelam que o coeficiente que representa a relação linear é menor do que a unidade. Uma indicação de que as oportunidades de crescimento industrial, no que diz respeito a geração de emprego, não se comportam de forma homogênea entre os diferentes portes das indústrias gaúchas, quando usa-se o número de empregados como medida.

Por seu turno, o modelo não-linear apresenta um coeficiente do termo quadrático que é negativo, indicando que a distribuição dos dados de emprego é côncava. Sendo assim, sua evolução neste setor, não foi acompanhada pela concentração do emprego nas maiores empresas, e nem ocorreu uma proliferação de empresas de porte micro. Na verdade, são as empresas de porte intermediário, as pequenas e as médias, que podem estar representando a dinâmica do emprego do setor no período de análise.

Apesar dos resultados aqui encontrados serem úteis para caracterizar o processo de Divergência e de distribuição da indústria gaúcha, há diversos aspectos exógenos que podem contribuir para melhor explicar esses fenômenos. Salienta-se que o modelo de Krugman (1997), aqui utilizado, falha em identificar as diferenças de estrutura de mercado, bem como os fatores que geram a divergência.

Como proposta futura de pesquisa, sugere-se a aplicação de uma estrutura microeconômica que relacione a variável de Divergência aqui aplicada, com outras determinantes, como renda das regiões, custos de transporte, disponibilidade de infra-estrutura, acesso a mão-de-obra, tecnologia dentre outros fatores. A partir do uso de ferramentas econométricas, será possivel gerar elasticidades que irão ajudar na interpretação do padrão de deslocamento das indústrias entre as regiões, seja de um Estado ou então de um País, da mesma forma que as decisões de investimento empresarial. 


\section{Referências}

ALONSO, J.A.F.; BENETTI, M.D.; BANDEIRA, P.S. Crescimento econômico da Região Sul do Rio Grande do Sul: causas e perspectivas. Porto Alegre: FEE, 1994.

ALVES, T.W. Fatores do crescimento regional agropecuário no Rio Grande do Sul 1970-96. Ensaios FEE, Porto Alegre, v. 27, n. 1, p. 233-272, maio 2006.

AUDRESCHT, D.; FELDMAN, M. RED spillovers and the geography of innovation and production, The American Economic Review, v. 86, n. 3, 1996.

AXTELL, R.L. Zipf's distribution of U.S. firm sizes. Science 293, p.1818-1820, 2001.

BAUMONT, C.; ERTUR, C.; LE GALLO, J. Geographic spillover and growth: a spatial econometric analysis for European Regions, LATEC - Document de travail, Economic, p. 2000-07, 2000.

BLACK, D.; HENDERSON, V. Urban evolution in the USA, Journal of Economic Geography, n. 3, p. 343-372, 2003.

BRAKMAN, S.; H. GARRETSEN, C. VAN MARREWIJK; M. van den Berg, The Return of Zipf's: Towards a further understanding of the Rank-Size Distribution, Journal of Regional Science, v. 39, n. 1, p. 183-213, 1999.

BRAKMAN, S.; GARRETSEN, H.; MARREWIJK, C.V. An introduction to geographical economics, Cambridge University Press, 2005.

BRÜLHART, M. Growing alike or growing apart? Industrial specialization of EU countries, In:WYPLOSZ, C. (ed.) The impact of EMU on Europe and the Developing Countries, Oxford University Press, 2001.

CAVALCANTE, L.R.M.T. Produção teórica em economia regional: uma proposta de sistematização, NPGA, UFBA, 2005.

DELGADO, A.P.; GODINHO, I.M. The evolution of city size distribution in Portugal: 1864-2001, Working Paper. CEDRES - Centro de Economia e Desenvolvimento Regional, Estudos e Serviços, Universidade do Porto, 2004.

DOBKINS, L.H.; IOANNIDES, Y.M. The evolution of size distribution: U.S. cities, in Economics of Cities, In: Theoretical Perspectives, J.M. Huriot and J.F. Thisse (eds), Cambridge, UK: Cambridge University Press, 2000.

ELLISON, G.; GLAESER, E. Geographic concentration In: U.S. manufacturing industries: a dartboard approach, Journal of Political Economy, n. 105, v. 5, p. 889-927, 1997.

FEE. Um século de população do Rio Grande do Sul, CD-ROM, Porto Alegre, 2001. . PIB municipal do RS 1985-01, CD-ROM, Porto Alegre, 2003.

FONSECA, P.S.D. A integração do Norte do Rio Grande do Sul à economia Gaúcha, Perspectiva Econômica, São Leopoldo, v. 10, n. 29, p. 21-36, 1980.

. A transição capitalista no Rio Grande do Sul: A economia gaúcha na República Velha. Estudos Econômicos, Instituto de Pesquisas Econômicas, 1984.

. Os gaúchos e a perspectiva nacional - das fazendas ao catete, In: Gonzaga, Sergius (Org.). Nós, os gaúchos. Porto Alegre, Editora da UFRGS, p. 157-161, 1992. 
. O processo de substituição de importações. In: Rego, José Márcio; Marques, Rosa Maria. (Org.). Formação Econômica do Brasil. São Paulo, SP: Editora Saraiva, p. 248-282, 2003.

FUJITA, M.; KRUGMAN, P.; VENABLES, A. The spatial economy. MIT Press, 1999.

GABAIX, X.; IOANNIDES, Y.M. - The Evolution of City Size Distributions, Working Paper Tufts University. Disponível em: http://ase.tufts.edu/econ, 2003.

GIAMBIAGI, F.; CASTRO, L.B.; HERMANN, J. Economia Brasileira Contemporânea (19452004). Ed. Campus, 2004.

HADDAD, P.R. Medidas de localização e de especialização. In: HADDAD, P.R. et al. (Org.). Economia regional teorias e métodos de análise, Fortaleza, BNB-ETENE, 1989.

HILL, B.M. A simple general approach to inference about the trial of a distribution. Annals of Statistics, v. 3, n. 5; p. 1163-1174, 1975.

KRUGMAN, P.R. The self-organizing Economy. Blackwell Publishers, Oxford, 1996. . Geography and Trade. MIT Press, 1997

LAGE, F.L. A localização da indústria de transformação brasileira nas últimas três décadas. 2002. Dissertação de Mestrado - EPGE/FGV, Rio de Janeiro.

LAUTERT, V. A dinâmica da concentração geográfica da indústria no Rio Grande do Sul: 1872 a 2000, Ensaios FEE, Porto Alegre, v. 26, nº especial, p. 37-62, 2005.

MADARIAGA, N.; MONTOUT, S.; OLLIVAUD, P. Regional Convergence and Agglomeration in Argentina: a spatial panel data approach, Working Paper, 2005.

MARQUES, H. The "New" economic theories. Working Paper, n. 104, Faculdade de Economia, Universidade do Porto, 2001.

MIDELFART-KNARVIK, K.H.; OVERMAN, F.G.; REDDING,S.J.; VENABLES, A.J. The location of European Industry, Economic Papers, n. 142, Report prepared for the directorate general for economic and financial affairs, European Comission, 2000.

MONASTERIO, L. M. A lei de Zipf: Rio Grande do Sul (1940-2000). II Encontro de Economia Gaúcha, 2004.

MORAIS, I.A.C.; SILVA, A.B.M.E. Crescimento econômico e populacional em cidades gaúchas, In: X encontro de economia da região Sul, Anpec-Sul, Porto Alegre, v. 1, 2007.

NISHIYAMA, Y.; OSADA, S. Statistical theory of rank-size rule regression under pareto distribution, Kyoto university discussion paper. Disponível em: http://www.kier.kyotou.ac.jp/coe21/dp/01-10/DP009-nishiyamaE3oasada.pdf, 2004.

OKUYAMA, K.M.; H. TAKAYASU. Zipf's Law in income distribution of companies, Physica A, n. 269, p. 125-131, 1999.

PACHECO, C.A. Novos padrões de localização industrial? Tendências recentes dos indicadores da produção e do investimento industrial, Brasília, IPEA, Texto para discussão, n. 633, 1999.

PORTUGAL, M.S. As políticas brasileiras de comércio exterior - 1947-88, Ensaios FEE, v. 1, n. 15: 234-252, 1994.

RESENDE, M.; WYLLIE, R. Aglomeração industrial no Brasil: um estudo empírico, Texto para discussão, IE-UFRJ, 2004. 
ROSEN, K.; RESNICK, M. The size distribution of cities: an examination of the Pareto law and primacy, Journal of Urban Economics, n. 8, p. 165-186, 1980.

SOO, K.T. Zipf's law for cities: a cross country investigation, CEP discussion paper, n. 641, 2004.

STÜLP, V.J. A evolução regional da produtividade da mão-de-obra no setor agropecuário do Rio Grande do Sul, Ensaios FEE, Porto Alegre, v. 27, n. 2, p. 377-410, out 2006.

SUZIGAN, W.; FURTADO, J.; GARCIA, R.; SAMPAIO, S.E.K. Aglomerações industriais no Estado de São Paulo, Economia Aplicada, n. 5, p. 695-717, 2001.

SUZIGAN, W.; FURTADO, J.; GARCIA, R.; SAMPAIO, S.E.K. Coeficientes de Gini locacionais - GL: aplicação à indústria de calçados do Estado de São Paulo, Nova Economia, v. 13, n. 2, p. 39-60, 2003.

VIEIRA, E.F.; RANGEL, S. Geografia econômica do Rio Grande do Sul: espacialidade/ temporalidade na organização econômica riograndense, Porto Alegre: Sagra e Luzzatto, 1993. $160 \mathrm{p}$.

ZIPF, G. K. Human behavior and the principle of least effort. Addison-Wesley, Cambridge, MA, 1949. 